\title{
Dementia With Lewy Bodies: the Principles of Diagnostics, Treatment, and Management
}

\author{
Jūratė Macijauskiené, Vita Lesauskaitė \\ Department of Geriatrics, Medical Academy, Lithuanian University of Health Sciences, Lithuania
}

\begin{abstract}
Key words: dementia with Lewy bodies; dementia syndrome; diagnostics; treatment; management.

Summary. Dementia with Lewy bodies was first recognized as a separate entity about 30 years ago. The prevalence varies from $0 \%$ to $5 \%$ in the general population, and this disease accounts for $0 \%$ to $30.5 \%$ of all dementia cases. Dementia with Lewy bodies is considered the second most common cause of degenerative dementia after Alzheimer's disease. The disease is characterized by alpha-synuclein immunoreactive protein deposits in both neurons and glial cells. The protein deposits are especially prominent in dopaminergic neurons, where they can be detected using conventional histological stains, such as hematoxylin and eosin, and are commonly referred to as Lewy bodies. The diagnosis of dementia with Lewy bodies is based on the presence of dementia as well as 2 of the following 3 core diagnostic features: 1) fluctuating cognition, 2) visual hallucinations, and 3) movement disorder. Diagnostic tests include laboratory data, structural and functional imaging, and electroencephalography. Differential diagnosis of dementia with Lewy bodies focuses on other later life dementia syndromes, other parkinsonian diseases (Parkinson's disease, progressive supranuclear palsy, corticobasal degeneration), and primary psychiatric illnesses. There is type $1 b$ evidence to support treatment with cholinesterase inhibitors. Glutamatergic and dopaminergic therapies are used as well. Standard neuroleptics are contraindicated, and atypical agents should be used cautiously. Nonpharmacologic measures - therapeutic environment, psychological and social support, physical activity, behavioral management strategies, caregivers' education and support, and different services - could be suggested.
\end{abstract}

\section{Introduction}

Dementia with Lewy bodies (DLB) was first recognized as a separate entity about 30 years ago; now, it is recognized being the second or third most common neurodegenerative dementia after Alzheimer's disease (AD).

Dr. Kenji Kosaka, a Japanese psychiatrist, with colleagues in 1976 described the concept of DLB for the first time (1). Two years later, Kosaka reported the distribution patterns of Lewy bodies (LBs) in cerebral cortex in 3 autopsied cases (2).

Historically, DLB has been given a variety of names, such as diffuse Lewy body disease, Lewy body dementia, Lewy body variant of Alzheimer's disease, Alzheimer's disease with incidental Lewy bodies, senile dementia of Lewy body type, and dementia associated with cortical Lewy bodies. Other dementias, such as AD, Parkinson's disease with late dementia, progressive supranuclear palsy, vascular dementia, and rare Creutzfeldt-Jacob disease, clinically can be difficult to distinguish from DLB (3). Neuropathologically, widely spread Lewy bodies in

Correspondence to J. Macijauskiene, Department of Geriatrics, Medical Academy, Lithuanian University of Health Sciences, A. Mickevičiaus 9, 44307 Kaunas, Lithuania

E-mail: jurate.macijauskiene@1smuni.lt cortex with or without plaques characteristic of AD were described (4).

The incidence of late-onset neurodegenerative illnesses associated with dementia is rapidly increasing due to the well-known demographic aging of the populations across the world with considerable clinical and socioeconomic consequences. Due to this, dementia syndrome and its causes are extensively studied, although its etiology remains unknown, and efficient treatment strategies are being developed. It is estimated that about 3 million Europeans will be affected by DLB by the year 2020, if no efficient treatment is offered (3).

This article reviews the current concept of the disease, clinical criteria, diagnostic methods, and management of DLB.

\section{Epidemiology}

Zaccai and colleagues performed a systematic review on the prevalence and incidence of DLB (5). In this review, of the 221 articles retrieved from MEDLINE, 7 studies met the inclusion criteria - original studies that used the McKeith 1996 criteria (one study used the 1995 criteria with no restriction on the duration of Parkinson's disease) for DLB and the DSM III and IV criteria for dementia. Prevalence 
estimates for DLB varied from $0 \%$ to $5 \%$ with regard to the general population and from between $0 \%$ to $30.5 \%$ of all dementia cases (5). According to Weisman and McKeith, if the upper limit of DLB prevalence is correct, then DLB is the second most common form of dementia after AD, replacing vascular dementia (6). McKeith et al. highlights the prevalence of DLB being the second most common cause of degenerative dementia after AD (7). The estimates for DLB prevalence reported for true population-based studies are generally lower than those from other types of samples. Prevalence rates reported in studies by neurologists $(0 \%-2.8 \%)$ were different from those reported by other specialists, such as psychiatrists and geriatricians $(3.6 \%-30.5 \%)$ (5). It may be due to a different emphasis on clinical phenotypes while diagnosing DLB: psychiatrists pay more attention to psychiatric symptoms and neurologists to neurological ones. Another explanation could be that in some patients with a diagnosis of DLB, Parkinson disease (PD), which is clinically allied to DLB, should have been diagnosed. The recent meta-analysis from China has reported the prevalence of DLB ranging from $0 \%$ to $0.03 \%$ in the population (8).

There are very few studies on the incidence of DLB. Miech and colleagues found that the incidence of DLB was $0.1 \%$ per year for the general population and $3.2 \%$ per year for all new cases of dementia (9).

\section{Neuropathology and Neurochemistry}

DLB is characterized by the deposits of alphasynuclein immunopositive protein in neuronal cells (10). The protein deposits are especially prominent in the dopaminergic neurons of the substantia nigra, where they are easily detected as eosinophilic inclusions stained with traditional histological stains (11). Lewy and Forster originally described Lewy inclusions in 1912 in the dorsal motor nucleus of the vagal nerve in cases of PD (3).

These eosinophilic neuronal inclusions, called Lewy bodies, made of proteins derived from neurofilaments, alpha-synuclein, and ubiquitin may occur in brain stem nuclei, cerebral cortex, and limbic system $(11,12)$. Immunochemistry for alpha-synuclein is a recommended method for the detection of LBs (12).

The normal function of alpha-synuclein is not well known; it is associated with synaptic vesicles, although does not play major role (13). A mutation may predispose alpha-synuclein to aggregate or interact abnormally with other proteins and provoke the formation of LBs (14). Alpha-synuclein is also associated with other disorders, such as multiple system atrophy, Pick's disease, and other neurodegenerations (15), which suggests that DLB is part of a larger group of neurode- generative disorders so-called synucleinopathies.

Beta-synuclein inhibits alpha-synuclein aggregation that is essential in the pathogenesis of Lewy body diseases in vitro and in vivo (16). An obvious decrease in beta-synuclein was observed in the cortical areas of the samples that corresponded neuropathologically to pure diffuse Lewy body pathology and clinically to DLB, differently from diffuse Lewy body pathology and concomitant Alzheimer's disease pathology or PD with dementia (16).

The topography of alpha-synuclein accumulation is used to define the type of LB disease (3). The limbic cortex, subcortical nuclei, and the brainstem are common sites of LBs in DLB. The neuropathological verification of DLB requires the assessment of the frontal, temporal, and parietal neocortex, the limbic and paralimbic regions, including the anterior cingulated area, and the brainstem (3). The accumulation of alpha-synuclein in glia is a pathological hallmark in DLB (17).

\section{Clinical Manifestations and Diagnostic Work-up}

Timely and accurate diagnosis is very important due to several reasons: 1) neuroleptic sensitivity; 2) effectiveness of the treatment with cholinesterase inhibitors; and 3) some patients may respond to dopaminergic treatment of parkinsonism.

The first consensus guidelines for the clinical diagnosis of DLB were introduced in 1996 (18) and later revised in 2005 (7). Table presents the key diagnostic criteria.

Briefly, a standard diagnosis of DLB is based on the presence of dementia as well as 2 of the following 3 core diagnostic features: 1) fluctuating cognition; 2) visual hallucinations; and 3) movement disorder.

It is estimated that $50 \%-75 \%$ of individuals with DLB experience fluctuations in cognition with pronounced variation in attention and alertness, varying over minutes, hours, or days, and excessive daytime drowsiness with transient confusion (misdiagnosis of transient ischemic attack) (19). The fluctuations of DLB resemble the signs of delirium without identifiable triggers. More than two-thirds $(70 \%)$ of individuals will develop akinetic-rigid syndrome (20). Tremor, bradykinesia, and rigidity tend to be more symmetric than asymmetric, and tremor tends to be maximal with posture/action rather that at rest (19).

Supportive features include syncope, postural instability with repeated falls, transient loss of consciousness, delusions, and multimodal hallucinations (i.e., visual and tactile) (6). Visual hallucinations occur during the course of illness in 13\%-80\% of all patients (7) and consist of fully formed, detailed, and 3-dimensional objects, provoking response, people, or animals that are not attributable 
Table. Diagnostic Criteria for Dementia With Lewy Bodies (7)

1. Central features for a diagnosis of possible or probable dementia with Lewy bodies

- Dementia defined as progressive cognitive decline of sufficient magnitude of interfere with normal social and occupational function

- Prominent or persistent memory impairment may not necessarily occur in the early stages but usually is evident with progression

- Deficits on tests of attention, executive function and visuospatial ability may be especially prominent

2. Core features ( 2 sufficient for probable dementia with Lewy bodies or 1 for possible dementia with Lewy bodies)

- Fluctuating cognition with pronounced variation in attention and alertness

- Recurrent visual hallucinations that typically are well formed and detailed

- Spontaneous features of Parkinsonism

3. Suggestive features

- REM sleep behavior disorder (RBD)

- Severe neuroleptic sensitivity

- Low dopamine transporter uptake in basal ganglia demonstrated by single-photon emission CT or positron emission tomographic imaging

Reprinted with permission from Lippincott, Williams \& Wilkins.

to perceptual distortion or illusion (19). This may be associated with greater deficits in cortical acetylcholine and predict better response to cholinesterase inhibitors, but other neurotransmitters, including dopamine and serotonin, have a contributory role (21). Auditory hallucinations rarely occur in patients who do not have visual hallucinations.

Based on the consensus criteria (7), if one or more of suggestive features is present in the presence of one or more core features, a diagnosis of probable DLB can be made. In the absence of any core features, one or more suggestive features are sufficient for possible DLB. Probable DLB should not be diagnosed based on suggested features alone.

Neuroleptic sensitivity, as a feature, is more important for the treatment than for establishing a diagnosis.

DLB is typically associated with cognitive complaints rather than aphasia or other language disturbances early in the course of the disease (22). Cognitive impairment in DLB is related to severe memory impairment, attentional deficits, visuospatial dysfunction, prosopagnosia, color agnosia, constructional and ideomotor apraxia (19, 22-24). Language disturbance in DLB is characterized by confabulation, incoherence, and perseveration during conversation, difficulty naming common objects, and a reduction in verbal fluency $(22,23)$.

Symptoms of the disease progress with deteriorating motor function, severe memory disturbance, neuroleptic reactions, disproportionate visuospatial deficits, and global functioning deterioration in late stages $(22,24)$.

Age at onset of DLB is similar to AD, with a mean of 68 years (range, 50-85 years) (25).

The diagnostic algorithm for DLB is analogous for a diagnostic process for other dementias. History taking and comprehensive physical and mental examinations are essential for the diagnosis. Laboratory studies include usual tests for the evaluation of dementia: complete blood count, chemistry panel, thyroid hormones, vitamin $\mathrm{B}_{12}$ level, and other tests when appropriate.

Structural imaging (computed tomography [CT], magnetic resonance imaging [MRI]) reveals the preservation of the medial temporal lobe, hippocampus, amygdale and atrophy of putamen (26). Functional imaging includes single-photon emission tomography (SPECT) and positron emission tomography (PET). On SPECT, parieto-occipital hypoperfusion (27) is seen for patients with DLB. PET shows hypometabolism - a reduction in striatal uptake of a ligand for the presynaptic dopamine transporter site (28). Hypermetabolism in some brain areas can be associated with the occurrence of visual hallucinations in patients with DLB (29). Hyperperfusion in deep gray matter is detected by iodine $123\left({ }^{123} \mathrm{I}\right) \mathrm{N}-$ isopropyl-p-iodo-amphetamine SPECT (30). Sensitivity and specificity of SPECT are considered to be around $60 \%-65 \%$, but an axial (123I)-2b-carbomethoxy-3b-(4-iodophenyl)-N-(3-fluoropropyl) nortropane ( ${ }^{123} \mathrm{I}-\mathrm{FP}-\mathrm{CIT}$ ) (dopaminergic) SPECT scan has a sensitivity of $78 \%$ and a specificity of $90 \%$ with an overall accuracy of $86 \%$ (31).

Other instrumental investigations can be useful supporting the diagnosis of DLB. Cardiovascular autonomic dysfunction is particularly common in DLB, and scintigraphy with ${ }^{123}$ I-metaiodobenzylguanidine (MIBG) allows the quantification of postganglionic cardiac sympathetic innervation (32). Cardiac scintigraphy with MIBG shows a reduced cardiac uptake of MIBG. Yoshita with coworkers revealed that the cutoff value of the heart-to-mediastinum ratio of 1.68 yielded a sensitivity of $100 \%$ and a specificity of $100 \%$ for differentiating DLB from AD (32). Other study has recently reported that the cutoff value of the heart-to-mediastinumratio of 1.36 differentiated DLB from other dementias with a sensitivity of $94 \%$ and a specificity of 96\% (33). Kobayashi with colleagues identified that DLB patients with orthostatic hypotension had lower heart-to-mediastinum ratios compared with those 
without orthostatic hypotension that implies severe impairment of the cardiac sympathetic nerves with clinically evident orthostatic hypotension in DLB patients (34). However, pathological MIBG findings is difficult to be interpreted, since diabetes and myocardial infarction can damage postganglionic sympathetic neurons, which may lead to false-positive MIBG findings (32).

An increase in an oxidized alpha-helical form of amyloid $\beta_{40}$ (interaction of amyloid beta and alphasynuclein) (35) and lower levels of alpha-synuclein (36) are found in the cerebrospinal fluid (CSF). Reduced levels of CSF alpha-synuclein in DLB may reflect the accumulation of alpha-synuclein with Lewy pathology in the brain, and the estimation of alpha-synuclein in combination with $\mathrm{A} \beta 42$ and tau in CSF can be used in the differentiation of DLB from AD and other dementias (37).

Electroencephalography (EEG) can show diffusely abnormal slowing of dominant rhythm, which according to the authors (38) may be related to a greater loss of choline acetyltransferase found in patients with DLB as compared with those with AD The loss of consciousness, which is characteristic of DLB, can be explained by temporal slow-wave transients revealed in EEG (38).

Genotyping shows mutations in the synuclein gene (39), APOE $\varepsilon 4$ allele (40), and leucine-rich repeat kinase 2 gene (Lrrk2). Although, the genotyping is inconclusive. Mutations in the synuclein gene can lead to the formation of LBs and a phenotype of DLB as well as PD, although this is not a major mechanism predisposing to DLB or PD. Lrrk2 mutations lead to the loss of dopaminergic neurons in the substantia nigra and are associated with inherited and sporadic PD, although mutations in the Lrrk2 not always manifest as LBs disease or synucleinopathy (41). A very recent study by Kobayashi and colleagues has reported APO $\varepsilon 4$ allele being a risk factor for DLB (42).

\section{Course of the Disease}

Some authors observed no difference in the rate of cognitive decline between DLB and AD (43). The others reported a shorter duration of illness for DLB compared with AD (44). DLB is associated with an increased risk of mortality, extrapyramidal signs being a strong predictor of mortality (45). Fluctuating dementia in early stage is a predictor of poor outcome (25). A higher institutionalization rate of patients with DLB compared with those with AD is documented (46).

\section{Differential Diagnosis}

A differential diagnosis of DLB focuses on the following: 1) other dementia syndromes in later life;
2) other parkinsonian diseases (Parkinson's disease, progressive supranuclear palsy, and corticobasal degeneration); and 3) primary psychiatric illnesses.

$A D$ is the Most Common Misdiagnosis. Clinically, at early stage, DLB and AD are similar, and before autopsy, many patients with DLB are diagnosed with $\mathrm{AD}$ (47). In DLB, in the early stages of the disease, fluctuating cognition is pronounced (3), although being not easily recognized (7). As reported by Calderon with colleagues, patients with DLB showed a significantly greater impairment in attention, working memory, and visuoperceptual ability than patients with AD despite the severity of dementia (48). Hallucinations tend to occur early in DLB course, whereas they tend to occur in the advanced stages of $\mathrm{AD}$ (44). Biomarkers are becoming important discriminating tools in the differential diagnosis of neurodegenerative dementia. Kasuga et al. demonstrated (37) that CSF alpha-synuclein levels in DLB patients were lower than those in patients with AD and other dementias. They also showed that levels of total and phosphorylated tau as well as $\mathrm{A} \beta 40 / 42$ and tau/A $\beta 42$ ratios in $\mathrm{AD}$ patients were different from those in patients with DLB and other dementias.

Parkinson's Disease with Dementia. Histopathological investigations of both Parkinson's disease with dementia (PDD) and DLB revealed the presence of large alpha-synuclein protein deposits (LBs) in the brain (49). There is an ongoing discussion whether PDD and DLB are two distinguishable disorders or share a common etiology $(50,51)$. The current available knowledge does not allow for a definite opinion. Thus, currently PDD and DLB are recognized as a subgroup of disorders termed synucleinopathies (51). LBs accumulate within the neuronal cytoplasm, destructuring neuronal cells. In both PDD and DLB, neurons due to accumulation of LBs are lost within the substantia nigra, a midbrain structure producing dopamine (52), although in DLB, LBs are more numerous in the cerebral cortex (22). PDD and DLB share environmental factors such as exposure to pesticides, high soil concentrations of specific metals (i.e., manganese) and others (53). Because of similar underlying neuropathology, the clinical pictures of PDD and DLB considerably overlap. Late-stage PDD may be similar to DLB, and DLB with progression is characterized by notable parkinsonism $(22,54)$. DLB is typically associated with greater memory loss and greater fluctuations in attention than PDD at early stages. Emre with colleagues did not report significant differences between these two groups in deficits of executive function, constructional praxis, and visuospatial processing (55). Difficulty in distinguishing between DLB and PDD is tend to be alleviated with a "one-year rule": dementia preceding the onset of a movement disorder by one year is classified as DLB, whereas dementia that occurs in the presence of ex- 
isting movement disorder is classified as PDD (55). Although, the rule remains controversial and is suggested to be used in scientific studies (7).

\section{Treatment and Management Pharmacologic Treatment}

There are currently no disease-modifying agents for the treatment of any neurodegenerative dementias. However, symptomatic treatment is available for the cognitive, psychiatric, and motor symptoms of DLB.

Transmitter Replacement Therapy With Acetylcholinesterase Inhibitors. Type $1 \mathrm{~b}$ evidence supports treatment with cholinesterase inhibitors in DLB, both dementia with LBs and Parkinson's disease dementia (including for neuropsychiatric symptoms) (56). Acetylcholinesterase inhibitors (donepezil and rivastigmine) are prescribed with the aim to treat cognitive symptoms of DLB, but these drugs may also be useful for the alleviation of psychiatric and motor symptoms (57). Effectiveness of cholinergic drugs is explained by a cholinergic deficit existing in DLB, which impairs the level of consciousness, awareness, and thus the sleeping and daytime activity cycle (57). Thus, anticholinergic drugs should improve consciousness. Titration to a low dose of donepezil (1.5 mg) might be useful in some DLB patients who show hypersensitivity to the standard dose of donepezil (58). A study by McKeith and colleagues that included 120 patients with DLB showed that treatment with rivastigmine at a dose of 6-12 $\mathrm{mg}$ during 20 weeks resulted in a significant clinical improvement in about half of the patients (59).

Memantine. Altered glutamatergic markers have been identified in patients with DLB (60), providing a rationale for therapy with $\mathrm{N}$-methyl D-aspartate receptor antagonist memantine for these individuals. Aarsland and colleagues reported that the patients who received memantine improved more than the patients who received placebo (61). In the recent study, Emre and colleagues reported (62) a mild benefit of memantine in terms of the overall clinical status and behavioral symptoms in patients with DLB, and memantine was more beneficial for patients with DLB than for patients with PDD. However, no meta-analyses or systematic reviews on the use of memantine in the patients with DLB have been carried out to date.

Currently, there are no evidence-based guidelines available when the treatment with antidementia drugs should be discontinued. The expert group recommends the following procedure (51): neuropsychological testing should be repeated after 3 to 6 months, and withdrawal of antidementia medication should be considered if the deterioration of cognitive functions is documented. If a significant decrease in the cognitive function is observed after 3-year treatment, antidementia drugs should be discontinued.

Transmitter Replacement Therapy With Dopaminergic Therapies. Levodopa is very effective in Parkinson's disease, thus its potential effect in the treatment of patients with DLB has been studied as well. Levodopa is generally well-tolerated in DLB (63); it alleviates extrapyramidal signs and supposedly does not worsen psychotic symptoms significantly as seen in case of dopamine agonists or amantadine (47). There are suggestions that the levodopa-carbidopa response in DLB may be not so significant as in PD, but further studies are required (47). Bonelli with colleagues studied the effect of levodopa in patients with PD, PDD, and DLB and showed that motor improvement was similar in 3 groups studied, although the proportion of patients with an improvement of $10 \%$ and more was greater in PD than PDD and DLB (64). Dopaminergic treatment might be less effective for DLB patients due to several reasons: intrinsic striatal alpha-synuclein pathology, reduced caudal putamen dopamine D2 receptors, loss of dopamine D3 receptors on corticostriate projection neurones, and "nondopaminergic" motor features due to degeneration in other brainstem nuclei (63).

In patients with DLB, the movement disorder is treated if the symptoms interfere with function (51). Treatment with antiparkinsonian drugs should be started at the lowest possible dose and the dose should be increased cautiously, as visual hallucinations and delusions may be exacerbated or precipitated by the use of dopaminergic agents. Therapy with levodopa must be stopped if ineffective or it increases hallucinations (63).

Selegiline, an antiparkinsonian drug, which delays the nucleation phase of alpha-synuclein aggregation leading to the formation of nontoxic species (65), has a neuroprotective effect.

Treatment With Antipsychotics. Standard neuroleptics are contraindicated, since up to $50 \%$ of DLB patients treated may experience impaired consciousness, irreversible parkinsonism, or an autonomic dysfunction syndrome resembling the neuroleptic malignant syndrome (66). A study by Ballard in 1998 reported that severe neuroleptic sensitivity occurred only in DLB (compared with AD) and was seen in $29 \%$ of cases, in all cases occurring within 2 weeks' administration of a new neuroleptic or a dose change (67). No predictive value of age, gender, severity, or clinical profile was identified. The mechanisms are not understood yet, but it might be due to a failure to up-regulate D2 receptors (58). Atypical agents are not risk free but preferred when antipsychotics are necessary. Therapy with quetiapine can be started at a dose of 12.5-25 $\mathrm{mg}$ at bedtime and increased as needed (up to $200 \mathrm{mg}$ per day) (68). 
Olanzapine at doses of 5-10 mg per day is suggested as not worsening parkinsonism, but used cautiously due to an anticholinergic effect having a risk to exacerbate cognitive impairment (69). Olanzapine and quetiapine are useful treating delusions (70).

Clozapine can be prescribed at a dose of 6.25$12.5 \mathrm{mg}$ per day at bedtime and increased in 6.25-mg steps with weekly monitored blood count (71).

While prescribing the antipsychotics, one should acknowledge that agents with D2 antagonism exacerbate extrapyramidal signs and those with anticholinergic properties may worsen cognitive impairment (47).

For REM sleep behavior disorder, clonazepam $(0.25 \mathrm{mg})$ at bedtime, melatonin $(3 \mathrm{mg})$ at bedtime, or quetiapine $(12.5 \mathrm{mg})$ at bedtime can be suggested (72).

For depression and anxiety, selective serotonin reuptake inhibitors (citalopram, escitalopram, sertraline, paroxetine, etc.) or serotonin and noradrenaline reuptake inhibitors (venlafaxine, mirtazapine, and bupropion) are the first-line agents; tricyclic antidepressants should be avoided because of their well-known anticholinergic side effects. There is an opinion that electroconvulsive therapy plays a role in the treatment of DLB, particularly for refractory depression, but also for psychosis and parkinsonism (73). Further research is needed.

\section{Nonpharmacologic Management}

Nonpharmacologic measures are of extreme importance since patients with DLB are at the highest risk for complications of drug therapy. Nonpharmacologic measures are the same as for other dementia syndromes: therapeutic environment, psychological and social support, physical activity, behavioral management strategies, education and support of caregivers, and support services. Clinical management of DLB includes the education of patients and caregivers about symptoms and expectations of the progression of the disease and needs for future care (50). As in cases of all dementias, a patient with

\section{References}

1. Kosaka K, Oyanagi S, Matsushita M, Hori A, Iwase S. Presenile dementia with Alzheimer-, Pick- and Lewy-body changes. Acta Neuropathol 1976;36:221-33.

2. Kosaka K. Lewy bodies in cerebral cortex, report of three cases. Acta Neuropathol 1978;42:127-34.

3. Graeber MB, Mueller U. Dementia with Lewy bodies: disease concept and genetics. Neurogenetics 2003;4:157-62.

4. Ince PG, Perry EK, Morris CM. Dementia with Lewy bodies. A distinct non-Alzheimer dementia syndrome? Brain Pathol 1998;8:299-324.

5. Zaccai J, McCracken C, Brayne C. A systematic review of prevalence and incidence studies of dementia with Lewy bodies. Age Ageing 2005;34:561-6.

6. Weisman D, McKeith I. Dementia with Lewy bodies. Semin Neurol 2007;27(1):42-7.
DLB is best served by a multidisciplinary team that may include the primary care and specialist physicians, nurses, and social workers. Problematic behaviors may be lessened with behavioral modification. Memory prompts, orientation, and attentional cues may be used to assist with memory deficits. Education of caregivers about psychiatric symptoms may reduce the need for use of antipsychotics. Modification of the environment may provide a better setting for persons experiencing visual hallucinations. Mobility aids may help with the motor symptoms of DLB. Some patients may benefit from short-term physical therapy. Patients and families should be educated about preparing their home for the prevention of falls and for safety in the kitchen. Patients should be advised to stop driving.

Caregivers of patients with DLB report unmet needs and challenges, such as social, medical, functional, psychological, and financial, expressing concerns about the future, feeling stressed, loss of social life, uncertainty, and "burnout" (74). Worse caregivers' situation is related to patients at risk of institutionalization and reduced quality of life (75). Support groups and caregivers' training can promote the emotional well-being of the caregiver and provide useful practical means of dealing with difficult situations.

\section{Problems and Future Investigations}

Future directions in research are focused on epidemiology of dementia with Lewy bodies in various populations, genetic background, improved understanding of pathophysiology, biomarkers and use of neuroimaging, interplay between genetic and environmental factors that lead to the clinical expression of the disease, monitoring of clinical course prospectively in a large and well-documented patients' cohort, improvement of clinical care, and diseasemodifying agents.

\section{Statement of Conflict of Interest}

The authors state no conflict of interest.

7. McKeith IG, Dickson DW, Lowe J, Emre M, O’Brien IT, Cummings J, et al. Diagnosis and management of dementia with Lewy bodies: third report of the DLB consortium. Neurology 2005;65:1863-72.

8. Dong M, Peng B, Lin X, Zhao J, Zhou Y, Wang R. The prevalence of dementia in the People's Republic of China: a systematic analysis of 1980-2004 studies. Age Ageing 2007;36(6):619-24.

9. Miech RA, Breitner JC, Zandi PP, Khachaturian AS, Anthony JC, Mayer L. Incidence of AD may decline in the early 90s for men, later for women: the Cache County study. Neurology 2002;58(2):209-18.

10. Londos E, Passant U, Gustafson L, Brun A. Neuropathological correlates to clinically defined dementia with Lewy bodies. Int J Geriatr Psychiatry 2001;16:667-79. 
11. Kövari E, Horvath J, Bouras C. Neuropathology of Lewy body disorders. Brain Res Bull 2009;80:203-10.

12. Spillantini MG, Schmidt ML, Lee VMY, Trojanowski JQ, Jakes R, Goedert M. Alpha-synuclein in Lewy bodies. Nature 1997;388:839-40.

13. Cookson MR. alpha-Synuclein and neuronal cell death. Mol Neurodegener 2009;4:9.

14. Trojanowski JQ, Lee VMY. Aggregation of neurofilament and alpha-synuclein proteins in Lewy bodies: implications for pathogenesis of Parkinson disease and Lewy body dementia. Arch Neurol 1998;55:151-2

15. Galvin JE, Lee VMY, Trojanowski JQ. Synucleinopathies. Arch Neurol 2001;58:186-90

16. Beyer K, Domingo-Sabat M, Santos C, Tolosa E, Ferrer I, Ariza A. The decrease of $\beta$-synuclein in cortical brain areas defines a molecular subgroup of dementia with Lewy bodies. Brain 2010;133:3724-33.

17. Terada S, Ishizu H, Yokota O, Tsuchiya K, Nakashima H, Ishihara $\mathrm{T}$, et al. Glial involvement in diffuse Lewy body disease. Acta Neuropathol 2003;105:163-9.

18. McKeith IG, Galasko D, Kosaka K, Perry EK, Dickson DW, Hansen LA, et al. Consensus guidelines for the clinical and pathologic diagnosis of dementia with Lewy bodies (DLB): report of the consortium on DLB international workshop. Neurology 1996;47:1113-24.

19. McKeith I, Mintzer J, Aarsland D, Burn D, Chiu H, CohenMansfield J, et al. Dementia with Lewy bodies. Lancet Neurology 2004;3(1):19-28.

20. Geser F, Wenning GK, Poewe W, McKeith I. How to diagnose dementia with Lewy bodies: state of the art. Mov Disord 2005;20(12):11-20.

21. McKeith IG, Wesnes K, Perry E, Ferrara R. Greater attentional deficits and responses to rivastigmine in dementia with Lewy body patients with visual hallucination. Dement Geriatr Cogn Disord 2004;18:94-100.

22. Reilly J, Rodriguez AD, Lamy M, Neils-Strunjas J. Cognition, language, and clinical pathological features of nonAlzheimer's dementias: an overview. J Commun Disord 2010;43:438-52.

23. Doubleday EK, Snowden JS, Varma AR, Neary D. Qualitative performance characteristics differentiate dementia with Lewy bodies and Alzheimer's disease. J Neurol Neurosurg Psychiatry 2002;72(5):602-7.

24. Ferman TJ, Smith GE, Boeve BF, Graff-Radford NR, Licas JA, Knopman DS, et al. Neuropsychological differentiation of dementia with Lewy bodies from normal aging and Alzheimer's disease. Clin Neuropsychol 2006;20(4):623-36.

25. Jellinger KA, Wenning GK, Seppi K. Predictors of survival in dementia with Lewy bodies and Parkinson dementia. Neurodegener Dis 2007;4(6):428-30.

26. Barber R, Ballard C, McKeith IG, Gholkar A, O'Brien JT. MRI volumetric study of dementia with Lewy bodies: a comparison with $\mathrm{AD}$ and vascular dementia. Neurology 2000;54(6):1304-9.

27. Shimizu S, Hanyu H, Kanetaka H, Iwamoto T, Koizumi K, Abe K, et al. Differentiation of dementia with Lewy bodies from Alzheimer's disease using SPECT. Dement Geriatr Cogn Disord 2005;20:25-30.

28. Lobotesis K, Fenwick JD, Phipps A, Ryman A, Swann A, Ballard C, et al. Occipital hypoperfusion on SPECT in dementia with Lewy bodies but not AD. Neurology 2001; 56:643-9.

29. Miyazawa N, Shinohara T, Nagasaka T, Hayashi M. Hypermetabolism in patients with dementia with Lewy bodies. Clin Nucl Med 2010;35:490-3.

30. Sato T, Hanyu K, Hirao S et al. Deep gray matter hypoperfusion on SPECT in dementia with Lewy bodies but not AD. Eur J Neur 2007;14:1299-1301

31. O'Brien JT. Role of imaging techniques in the diagnosis of dementia. Br J Radiol 2007;80:S71-S77.

32. Yoshita M, Taki J, Yokoyama K, Noguchi-Shinohara M, Matsumoto Y, Nakajima K, et al. Value of I-MIBG radioac- tivity in the differential diagnosis of DLB from AD. Neurology 2006;66:1850-4.

33. Estorch M, Camacho V, Paredes P, Rivera E, RodriguezRevuelto A, Flotats A, et al. Cardiac (123)I-metaiodobenzylguanidine imaging allows early identification of dementia with Lewy bodies during life. Eur J Nucl Med Mol Imaging 2008;35:1636-41.

34. Kobayashi S, Tateno M, Morii M, Utsumi K, Saito T. Decreased cardiac MIBG uptake, its correlation with clinical symptoms in dementia with Lewy bodies. Psychiatry Research 2009;74:76-80.

35. Bibl M, Mollenhauer B, Lewczuk P, Esselmann H, Wolf S, Trenkwalder C, et al. Validation of amyloid-beta peptides in CSF diagnosis of neurodegenerative dementias. Mol Psychiatry 2007;12:671-80.

36. Schlossmacher MG. Quantification of alpha-synuclein in dopamine cells, peripheral blood, genotyped brain and CSF. Neurodegener Dis 2007;4:187.

37. Kasuga K, Tokutake T, Ishikawa A, Uchiyama T, Tokuda $\mathrm{T}$, Onodera O, et al. Differential levels of alpha-synuclein, beta-amyloid42 and tau in CSF between patients with dementia with Lewy bodies and Alzheimer's disease. J Neurol Neurosurg Psychiatry 2010;81:608-10.

38. Briel RC, McKeith IG, Barker WA, Hewitt Y, Perry RH, Ince $P G$, et al. EEG findings in dementia with Lewy bodies and Alzheimer's disease. J Neurol Neurosurg Psychiatry 1999;66:401-3

39. Kurz MW, Larsen JP, Kvaloy JT, Aarsland D. Associations between family history of Parkinson's disease and dementia and risk of dementia in Parkinson's disease: a communitybased, longitudinal study. Mov Disord 2006;21(12):2170-4.

40. Singleton AB, Wharton A, O'Brien KK, Walker MP, McKeith IG, Ballard CG, et al. Clinical and neuropathological correlates of apolipoprotein $\mathrm{E}$ genotype in dementia with Lewy bodies. Dement Geriatr Cogn Disord 2002;14:167-75.

41. Santpere G, Ferrer I. LRRK2 and neurodegeneration. Acta Neuropathol 2009;117:227-46.

42. Kobayashi S, Tateno M, Park TW, Utsumi K, Sohma H, et al. Apolipoprotein E4 frequencies in a Japanese population with Alzheimer's disease and dementia with Lewy bodies. PLoS ONE 2011;6(4):e18569.

43. Helmes E, Bowler JV, Merskey H, Munoz DG, Hachinski VC. Rates of cognitive decline in Alzheimer's disease and disease with Lewy bodies. Dement Geriatr Cogn Disord 2003; 15:67-71.

44. Ferman TJ, Dickson DW, Graff-Radford N. Early onset of visual hallucinations in dementia distinguishes pathologically-confirmed Lewy body disease from AD. Neurology 2003;60(5):A264.

45. Williams MM, Xiong C, Morris JC, Galvin JE. Survival and mortality differences between dementia with Lewy bodies vs Alzheimer disease. Neurology 2006;67:1935-41.

46. Fujishiro H, Umegaki H, Suzuki Y, Isojima D, Akatsu H, Ikari $\mathrm{H}$, et al. Clinical profiles of autopsy-confirmed dementia with Lewy bodies at institutionalization: comparison with Alzheimer's disease. Psychogeriatrics 2007;7(3):98103.

47. Ferman TJ, Boeve BF. Dementia with Lewy bodies. Neurol Clin 2007;25:741-760.

48. Calderon J, Perry RJ, Erzlinclioglu SW, Berrios GE, DEnig TR, Hodges JR. Perception, attention, and working memory are disproportionally impaired in dementia with Lewy bodies compared with Alzheimer's disease. J Neurol Neurosurg Psychiatry 2001;70:157-64.

49. Theuns J, Van Broeckhoven C. Alpha-synuclein gene duplications in sporadic Parkinson disease. Neurology 2008; 70(1):7-9.

50. Lippa CF, Duda JE, Grossman M, Hurtig HI, Aarsland D, Boeve BF, et al. DLB and PDD boundary issues: diagnosis, treatment, molecular pathology, and biomarkers. Neurology 2007;68:812-9.

51. Dodel R, Csoti I, Ebersbach G, Fuchs G, Hahne M, Kuhn 
W, et al. Lewy body dementia and Parkinson's disease with dementia. J Neurol 2008;255(5):39-47.

52. Bear MF, Connors BW, Paradiso MA. Neuroscience: exploring the brain. 3rd ed. Philadelphia, PA: Lippincott Williams and Wilkins Publishers; 2007.

53. Ascherio A, Chen H, Weisskopf MG, O'Reilly E, McCullough ML, Calle EE, et al. Pesticide exposure and risk for Parkinson's disease. Ann Neurol 2006;60(2):197-203.

54. Byrne EJ, Lennox G, Lowe J, Godwin-Austen RB. Diffuse Lewy body disease: clinical features in 15 cases. I Neurol Neurosurg Psychiatry 1989;52(6):709-17.

55. Emre M, Aarsland D, Brown R, Burn DI, Duyckaerts C, Mizuno Y, et al. Clinical diagnostic criteria for dementia associated with Parkinson's disease. Mov Disord 2007; 22(12):1689-707.

56. Burns A, O'Brien J. Clinical practice with anti-dementia drugs: a consensus statement from British Association for Psychopharmacology. J Psychopharmacol 2006;20:732-75.

57. Simard M, van Reekum R. The acetylcholinesterase inhibitors for treatment of cognitive and behavioral symptoms in dementia with Lewy bodies. J Neuropsychiatry Clin Neurosci 2004;16:409-25.

58. Fujita Y, Takebayashi M. Efficacy of low-dose donepezil for visual hallucinations in a patient with dementia with Lewy bodies. Psychiatry Clin Neurosci 2010;64:336.

59. McKeith I, Del Ser T, Spano P, Emre M, Wesnes K, Anand $R$, et al. Efficacy of rivastigmine in dementia with Lewy bodies: a randomised, double-blind, placebo-controlled international study. Lancet 2000;356(9247):2031-6.

60. Dalfo E, Albasanz JL, Martin M, Ferrer I. Abnormal metabotropic glutamate receptor expression and signaling in the cerebral cortex in diffuse Lewy body disease is associated with irregular alpha-synuclein/phospholipase C(PLCbeta1) interactions. Brain Pathol 2004;14:388-9.

61. Aarsland D, Ballard C, Walker Z, Bostrom F, Alves G, Kossakowski K, et al. Memantine in patients with Parkinson's disease dementia or dementia with Lewy bodies: a doubleblind, placebo-controlled, multicentre trial. Lancet Neurol 2009;8(7):613-8

62. Emre M, Tsolaki M, Bonucelli U, Destée A, Tolosa E, Kutzelnigg A, et al. Memantine for patients with Parkinson's disease dementia or dementia with Lewy bodies: a randomized, double-blind, placebo-controlled trial. Lancet Neurol 2010;9:969-77.

63. Molloy S, McKeith IG, O'Brien JT, Burn DJ. The role of levodopa in the management of dementia with Lewy bodies. J Neurol Neurosurg Psychiatry 2005;76:1200-3.

64. Bonelli SB, Ransmayr G, Steffelbauer M, Lukas T, Lampl C, Deibl M. L-dopa responsiveness in dementia with Lewy bodies, Parkinson disease with and without dementia. Neurology 2004;63(2):376-8.

65. Braga CA, Follmer C, Palhano FL, Khattar E, Freitas MS, Romão L, et al. The anti-Parkinsonian drug selegiline delays the nucleation phase of $\alpha$-synuclein aggregation leading to the formation of nontoxic species. J Mol Biol 2011; 405:254-73.

66. McKeith I, Fairbairn A, Perry R, Thompson P, Perry E. Neuroleptic sensitivity in patients with senile dementia of Lewy body type. BMJ 1992;305:673-678.

67. Ballard C, Grace I, McKeith I, Holmes C. Neuroleptic sensitivity in dementia with Lewy bodies and Alzheimer's disease. Lancet 1998;351:1032-3.

68. Takahashi H, Yoshida K, Sugita T, Higuchi H, Shimizu T. Quetiapine treatment of psychotic symptoms and aggressive behavior in patients with dementia with Lewy bodies: a case series. Prog Neuropsychopharmacol Biol Psychiatry 2003;27:549-33.

69. Mulsant BH, Gharabawi GM, Bossie CA, Mao L, Martinez RA, Tune LE, et al. Correlates of anticholinergic activity in patients with dementia and psychosis treated with risperidone or olanzapine. J Clin Psychiatry 2004;65:1708-14.

70. Fischer C, Bozanovic R, Atkins JH, Rourke SB. Treatment of delusions in dementia with Lewy bodies - response to pharmacotherapy. Dement Geriatr Cogn Disord 2007;23: 307-11.

71. Burke WJ, Pfeiffer RF, McComb RD. Neuroleptic sensitivity to clozapine in dementia with Lewy bodies. J Neuropsychiatry Clin Neurosci 1998;10:227-9.

72. Boeve BF, Silber MH, Ferman TJ. REM sleep behavior disorder in Parkinson's disease and dementia with Lewy bodies. J Geriatr Psychiatry Neurol 2004;17:146-57.

73. Burgut FT, Kellner CH. Electroconvulsive therapy (ECT) for dementia with Lewy bodies. Med Hypotheses 2010;75: 139-40.

74. Galvin JE, Duda JE, Kaufer DI, Lippa CF, Taylor A, Zarit SH. Lewy body dementia: caregiver burden and unmet needs. Alzheimer Dis Assoc Disord 2010;24:177-81.

75. Etters L, Goodall D, Harrison BE. Caregiver burden among dementia patient caregivers: a review of the literature. I Acad Nurse Pract 2008;20:423-8.

Received 7 June 2011, accepted 29 January 2012 